\title{
Segurança do Trabalhador em Aplicações de Herbicidas com Pulverizadores de BarRa em CANA-De-AÇÚCAR ${ }^{1}$
}

\author{
Workers 'Safety in Herbicide Applications using Bar Sprayers in Sugar Cane
}

\author{
MACHADO NETO, J.G. ${ }^{2}$, COSTA, G.M. ${ }^{3}$ e OLIVEIRA, M.L. ${ }^{4}$
}

\begin{abstract}
RESUMO - Objetivou-se com este trabalho avaliar a eficiência da cabina do trator, de dois tipos de ponta de pulverização e de duas posições da barra do pulverizador montado em trator em aplicações de herbicidas na cultura de cana-de-açúcar, como medidas de proteção coletiva para a atividade de tratorista, separadamente ou combinadas; e classificar a segurança dessas condições de trabalho com as 46 recomendações de herbicidas registradas. As exposições dérmica e respiratória do tratorista foram quantificadas em aplicações com o pulverizador equipado com barra traseira ou central, associadas com pontas com indução de ar, modelo Turbo TeeJet Air Induction ${ }^{\circledR}$ (TTI-11004VP), e sem indução de ar, modelo Turbo Floodjet ${ }^{\circledR}$ (TF-VP3), e o trator sem e com cabina. Foram calculadas as margens de segurança (MS) para 46 recomendações de aplicação de herbicidas nessas condições de trabalho. Pelos valores de MS calculados, as condições de trabalho foram classificadas como seguras (MS $\geq 1$ ) ou inseguras ( $\mathrm{MS}<1$ ). A condição de trabalho mais segura para o tratorista é a associação de pulverizador de barra central, trator com cabina e pontas TTI. Nas aplicações com o pulverizador de barra central sem a cabina, das 46 recomendações de herbicidas, são seguras para o tratorista as de imazapyr, trifloxysulfuron-sodium, imazapic, glyphosate, amicarbazone, hexazinone, sulfentrazone, clomazone, oxadiazon, isoxaflutole, pendimethalin, flazasulfuron, tebuthiuron, ethoxysulfuron e acetoclor. Com o uso da cabina, também são seguras as de metribuzin e s-metalochlor. Nas aplicações com o pulverizador de barra traseira sem a cabina, das 46 recomendações de herbicidas, são seguras as de imazapyr, trifloxysulfuron-sodium, imazapic, glyphosate, amicarbazone, hexazinone, sulfentrazone, clomazone, oxadiazon, is oxaflutole, pendimethalin, flazasulfuron e tebuthiuron. Com a associação da cabina, também são seguras para o tratorista a aplicação do ethoxysulfuron com as pontas TF e a do metribuzin e s-metalochlor com as pontas TTI.
\end{abstract}

Palavras-chave: exposição dérmica, tratorista, ponta de pulverização, tamanho de gotas.

\begin{abstract}
This work was carried out to evaluate the efficiency of the tractor cabin, two nozzle models and two bar positions of a tractor-mounted sprayer in herbicide applications on sugar cane crop as a collective protection measure for tractor driver activity, separately or combined; and to classify the safety of these working conditions according to the 46 recommendations of registered herbicides. The truck driver's skin and respiratory organ exposures were quantified in applications with the sprayer equipped with back or central bar, nozzles with air induction, Turbo TeeJet Air Induction ${ }^{\circledR}$ (TTI-1 1004 VP) model and without air induction, Turbo Floodjet ${ }^{\circledR}$ (TF VP3) model, and tractor with and without cabin. The safety margins (SM) for 46 herbicide application recommendations under these work conditions were calculated. Based on the calculated MS

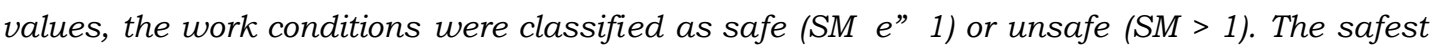
work conditions for the tractor driver were when the sprayer was combined with the central bar, tractor cabin and TTI model nozzles. With sprayer with central bar without cabin, of the 46
\end{abstract}

Recebido para publicação em 5.2.2007 e na forma revisada em 31.8.2007.

2 Prof. Adjunto do Dep. de Fitossanid ade da FCAV/UNESP - Jaboticabal, Via de Acesso Prof. Paulo Donato Castellane, 14844900. Jaboticabal-SP, <joaquim @ fcav.unesp.br>; ${ }^{3}$ Engo-Agro - Mestrando no Programa de Pós-Graduação em Agronomia - área de concentração em Produção Vegetal, da FCAV/UNESP, Jaboticabal-SP, <glauberto.moderno@ itelefonica.com.br>; ${ }^{4}$ Eng $^{\circ}-$ Agr $^{\circ}$, Dr., bolsista do CNPq, Dep. de Fitossanidade da FCAV/UNESP - Jaboticabal, Via de Acesso Prof. Paulo Donato Castellane, 14844900. Jaboticabal-SP, <oliveiraml@ hotmail.com>.

Planta Daninha, Viçosa-MG v. 25, n. 3, p. 639-648, 2007 
herbicide recommendations, imazapyr, trifloxysulfuron-sodium, imazapic, glyphosate, amicarbazone, hexazinone, sulfentrazone, clomazone, oxadiazon, isoxaflutole, pendimethalin, flazasulfuron, tebuthiuron, ethoxysulfuron and acetoclor were considered safe for the truck driver. With cabin use, metribuzin and s-metalochlor applications were also considered safe. In the applications with back bar sprayer and without the cabin, of the 46 herbicide recommendations, imazapyr, trifloxysulfuron-sodium, imazapic, glyphosate, amicarbazone, hexazinone, sulfentrazone, clomazone, oxadiazon, isoxaflutole, pendimethalin, flazasulfuron and tebuthiuron were considered safe for the truck driver. When combined with the truck cabin, applications of etosyulfuron with TF nozzles and of metribuzin and s-metalochlor with TTI nozzles were also considered safe for the truck driver.

Keywords: skin exposure, tractor driver, nozzle, droplet size.

\section{INTRODUÇÃO}

A grande maioria das aplicações de herbicidas na cultura de cana-de-açúcar é realizada com pulverizadores de barra montados em trator. Aplicar corretamente os agrotóxicos requer conhecimentos específicos para a correta colocação do produto no alvo, de forma a evitar a contaminação do ambiente de aplicação e do trabalhador. A parte dos agrotóxicos aplicados não retida no alvo, denominada de deriva, fica dispersa no ambiente e pode atingir organismos não-alvos e o trabalhador exposto. A deriva resulta em risco de intoxicação ambiental e do trabalhador exposto.

Definir modelos de pontas de pulverização que proporcionem redução da deriva, sem comprometer a cobertura mínima do alvo, é fundamental para prevenção da contaminação ambiental e da exposição do trabalhador. Menor deriva resulta em menores contaminações ambientais e exposições de organismos nãoalvos, e dos trabalhadores expostos (Coutinho et al., 2003). Quanto maior o diâmetro da gota, menor o risco de deriva e de evaporação da pulverização. Portanto, é imprescindivel que o pulverizador utilizado seja equipado com a ponta de pulverização adequada para produzir um espectro de gotas que proporcione o controle dos organismos-alvo com a menor quantidade eficiente do agrotóxico e a mínima deriva (Himel, 1969).

Entre os componentes do equipamento de aplicação, as pontas de pulverização estão entre as partes mais importantes do pulverizador na determinação do diâmetro das gotas da pulverização. Se selecionadas corretamente, definindo modelo, tamanho e pressão de trabalho, garantem a segurança ao meio ambiente e ao operador e a eficiência da aplicação (Gadanha Junior, 2000). O uso de pontas que produzem gotas grossas e com deriva reduzida - como é o caso das pontas com indução de ar - pode resultar em condições de trabalho mais seguras para os trabalhadores expostos.

Nas pulverizações de herbicidas em canade-açúcar com o pulverizador de barra, os tratoristas ficam expostos ao contato direto com a calda na forma de deriva ocorrida entre a ponta de pulverização e o alvo. Os herbicidas são tóxicos para os seres humanos e, portanto, os tratoristas ficam expostos a riscos de intoxicações. A intensidade do risco de intoxicação depende da exposição proporcionada pela condição de trabalho ao trabalhador e da toxicidade do agrotóxico em uso (Bonsall, 1985).

Em função do risco de intoxicação, as condições de trabalho podem ser classificadas em seguras ou inseguras. A segurança, ao contrário do risco, é representada pela probabilidade de não-ocorrência de lesões e intoxicações com agrotóxicos em virtude da exposição em condições específicas de trabalho (Machado Neto, 2001). Avaliar a segurança das condições de trabalho é fundamental para selecionar as medidas de segurança mais adequadas e suficientes para reduzir os riscos de intoxicações ocupacionais a níveis aceitáveis.

A condição de trabalho é composta pelo meio ambiente onde o trabalhador se encontra 
e pelos componentes materiais utilizados para realizar a sua atividade laboral. As medidas de segurança podem ser agrupadas em preventivas e de proteção, que, por sua vez, podem ser agrupadas em individuais e coletivas. As medidas de segurança coletiva são aquelas relacionadas ao meio e aos ambientes de trabalho para controlar a exposição dos trabalhadores. São dispositivos o sistema ou meio de abrangência coletiva destinado a preservar a integridade física e a saúde dos trabalhadores (ANVISA, 2006).

Dentre as medidas de segurança coletiva destacam-se as modificações nas estruturas das máquinas de aplicação de agrotóxicos, como alteração na posição, horizontal e/ou vertical, e na distância do trabalhador, em relação à pulverização, e que resultem em menores exposições (Machado Neto \& Matuo, 1992; Machado Neto et al., 1992). Nas aplicações de herbicidas na cultura de cana-de-açúcar, o risco de intoxicação dos tratoristas pode ser afetado por fatores como: tipo, tamanho e pressão da ponta de pulverização, posição da barra do pulverizador e uso de cabina no trator, entre outros. Esses fatores podem ser classificados como medida de proteção coletiva, ao reduzirem a exposição proporcionada pelas condições de trabalho ao trabalhador.

Para Gadanha Junior (2000), as características dos equipamentos modernos proporcionam razoável segurança e eficiência na aplicação dos agrotóxicos. Os pulverizadores de barra estão sendo equipados com pontas de pulverização antideriva, como as com indução de ar, mas ainda não se conhece o efeito dessa tecnologia sobre a redução da exposição proporcionada ao trabalhador e, principalmente, nos pulverizadores com barras localizadas no centro do trator.

Objetivou-se com este trabalho avaliar a eficiência da cabina do trator, de dois tipos de ponta de pulverização e de duas posições da barra do pulverizador montado em trator como medidas de segurança coletiva em aplicações de herbicidas na cultura de cana-de-açúcar para a atividade de tratorista, separadamente ou combinadas; e classificar a segurança dessas condições de trabalho com as 46 recomendações de herbicidas registradas.

\section{MATERIAL E MÉTODOS}

\section{Condições de trabalho do tratorista}

As avaliações das exposições dérmicas e respiratórias potenciais (EDPs e ERPs) proporcionadas aos tratoristas nas aplicações de herbicidas, na cultura de cana-de-açúcar com pulverizadores de barra, foram realizadas em duas fases, devido à disponibilidade dos equipamentos. A primeira fase foi realizada durante o mês de setembro de 2006 e a segunda, durante o mês de outubro do mesmo ano, ambas na região noroeste do Estado de São Paulo. As duas fases foram realizadas em áreas cultivadas com cana-de-açúcar, em condição de soqueira, em aplicações de herbicidas em pré e pósemergência inicial das plantas daninhas.

As aplicações foram feitas com quatro tratores dos seguintes modelos: Massey Ferguson, MF275, traçado, com cabina adaptada e outro sem cabina; Ford 6630 sem cabina; e John Deere cabinado. Os tratores trabalharam a $6,4 \mathrm{~km} \mathrm{~h}^{-1}$, em terceira marcha reduzida. O espaçamento dos bicos nas barras de pulverização foi de $64 \mathrm{~cm}$ e o volume de calda, de $200 \mathrm{~L} \mathrm{ha}^{-1}$.

O pulverizador de barra central utilizado foi do modelo Herbiplus, montado nos tratores MF275, com e sem cabina, e estava composto por tanque de 900 litros, bomba APS 96 (96 $\mathrm{L} \mathrm{min}^{-1}$ ), barra central com 13,5 m de comprimento e 18 bicos de pulverização.

Foram utilizados pulverizadores convencionais com barras traseiras de dois modelos: Condor 600, montado no trator Ford 6630, composto por tanque de 600 litros, bomba JP 75 (75 $\mathrm{L} \mathrm{min}^{-1}$ ), barra de $12 \mathrm{~m}$ e 18 bicos; e Falcon 800 , montado no trator John Deere 5600, composto por tanque de 800 litros, bomba JP 75 $\left(75 \mathrm{~L} \mathrm{~min}^{-1}\right)$, barra traseira com largura total de $14 \mathrm{~m}$ e 21 bicos.

Os dois modelos de pontas de pulverização utilizadas foram: convencionais sem indução de ar, de jato plano, modelo Turbo Floodjet (TFVP3) defletor; e com indução de ar, de jato plano, modelo Turbo TeeJet Air Induction (TTI11004VP). Ambas são denominadas de pontas antideriva, devido ao espectro de gotas extremamente grossas (Spraying Systems, 2006). 
As pontas TF operaram na pressão de $100 \mathrm{kPa}$ nas quatro condições de trabalho e produziram gotas com o valor do diâmetro mediano volumétrico (DMV) de $846 \mu \mathrm{m}$. As pontas TTI operaram na pressão de $200 \mathrm{kPa}$ nas quatro condições de trabalho e produziram gotas com o valor do DMV de $930 \mu \mathrm{m}$ (SprayingSystems, 2006).

Durante a aplicação dos tratamentos, a temperatura média variou entre 25,6 e $30,4^{\circ} \mathrm{C}$; a umidade relativa do ar, entre 44,7 e $71,9 \%$; e a velocidade do vento, entre 7,7 e $9,3 \mathrm{~km} \mathrm{~h}^{1}$.

\section{Quantificação das exposições dérmicas e respiratórias}

As avaliações das exposições dérmicas (EDs) e respiratórias (ERs) do tratorista foram realizadas em aplicações dos herbicidas na cultura de cana-de-açúcar. $O$ cátion manganês do sulfato de manganês $(31 \%$ de $\mathrm{Mn})$ foi utilizado como traçador nas caldas, de acordo com a metodologia adotada por Oliveira \& Machado Neto (2003a). O sulfato de manganês foi adicionado às caldas na dosagem de $250 \mathrm{~g}$ por $100 \mathrm{~L}$. As caldas das aplicações com o pulverizador de barra central foram compostas com os herbicidas Velpar K GRDA (diuron + hexazinone) a $2,0 \mathrm{~kg} \mathrm{ha}^{-1}$ e com o pulverizador de barra traseira, Metrimex SC (ametryn) 1,8 $\mathrm{L} \mathrm{ha}^{-1}$.

As exposições às caldas, determinadas com as quantificações desse cátion retido nas vestimentas amostradoras, foram utilizadas como dados substitutos para calcular as exposições aos herbicidas aplicados nas mesmas condições de trabalho, de acordo com Jensen (1984).

As avaliações das EDs e ERs foram feitas com dez repetições, e cada repetição da ER foi avaliada em tempos equivalentes a cinco repetições das EDs. As EDs e ERs foram extrapoladas para uma jornada diária de oito horas de trabalho. As EDs foram avaliadas com vestimentas amostradoras, compostas por um macacão de tecido de algodão com capuz, absorventes higiênicos femininos e luvas de algodão (Machado Neto \& Matuo, 1989; Machado Neto, 1997). Os absorventes higiênicos femininos $\left(\right.$ Carefree $\left.^{\circledR}\right)$ foram afixados sobre máscaras semifaciais descartáveis, para quantificar a exposição na face; na parte superior mediana dos pés, para quantificar a exposição dos pés; e nas luvas de algodão, para as mãos. As avaliações das exposições respiratórias (ERs) foram feitas com bombas com fluxo de ar contínuo de uso pessoal, da marca A.P. Buck, reguladas para succionar $2 \mathrm{~L}$ de ar por minuto, de acordo com a metodologia adaptada por Oliveira \& Machado Neto (2003b). Cada bomba foi equipada com uma mangueira plástica de sucção, contendo um cassete com filtro de éster celulose na extremidade.

Imediatamente após os períodos de exposição, os macacões foram seccionados, para representar as seguintes regiões do corpo do trabalhador: cabeça+pescoço, braços+antebraços, tronco-frente, tronco-atrás, coxas+pernasfrente e coxas+pernas-atrás.

A solubilização do cátion Mn nas partes seccionadas dos macacões, nos absorventes higiênicos femininos e nos filtros de éster celulose foi realizada com solução contendo $\mathrm{HCl}$ a 0,2 N (Oliveira \& Machado Neto, 2003a). As concentrações do traçador nos extratos, após filtragem em papel-filtro qualitativo, foram quantificadas em espectrofotômetro de absorção atômica, marca GBC, modelo 932AA. A partir da quantidade do $\mathrm{Mn}$ recuperada de cada absorvente higiênico feminino, que corresponde à ED na área do absorvente $\left(70 \mathrm{~cm}^{2}\right)$, fizeram-se as extrapolações para as respectivas áreas do corpo que representavam: pés ou face.

A curva-padrão do manganês foi realizada com cloreto de manganês ( $\mathrm{MnCl}$ ) Titrisol Merk 9988. A faixa de linearidade foi determinada com soluções aquosas nas concentrações de 0,125, 0,25, 0,5, 1,0; e 2,0 ppm $\left(\mathrm{R}^{2}=0,999\right)$. O LD foi de 0,017 ppm e o LQ, de 0,041 ppm. Os LDs e LQs foram calculados de acordo com Skoog et al. (1998).

Com os valores de $\mathrm{Mn}$ recuperados nos amostradores e a concentração do traçador nas caldas, determinada em amostras coletadas no campo para cada repetição das condições de trabalho, foram calculadas as EDs às caldas para cada região do corpo. As ERs às caldas foram calculadas com as quantidades de Mn recuperadas de cada filtro de éster celulose, a concentração do traçador pulverizado na calda a que os trabalhadores ficaram expostos e o tempo de amostragem do ar. A recuperação do cátion Mn no filtro de éster celulose foi 
previamente determinada em $92,0 \%$ por Oliveira (2000).

As EDs e ERs dos tratoristas, estimadas para os tempos de exposição em cada repetição, foram extrapoladas para um dia de trabalho com tempo de exposição efetiva de oito horas.

\section{Segurança das condições de trabalho}

A segurança das condições de trabalho do tratorista nas oito condições avaliadas, calculada para 46 recomendações das maiores dosagens (condições de maiores exposições) dos herbicidas para a cultura de cana-deaçúcar (Andrei, 2003; SIA, 2006), foi determinada por meio do cálculo da margem de segurança (MS), segundo Machado Neto (1997).

$$
\mathrm{MS}=\frac{\text { NOELXP }}{\text { QAEX FS }}
$$

em que:

NOEL = nível de efeitos não observados $\left(\mathrm{mg} \mathrm{kg}^{-1} \mathrm{dia}^{-1}\right)$;

$\mathrm{P}=$ peso corpóreo médio do trabalhador, considerado como $70 \mathrm{~kg}$;

$\mathrm{QAE}=$ quantidade absorvível da exposição $\left(\mathrm{mg} \mathrm{dia}{ }^{-1}\right) ; \mathrm{e}$

$\mathrm{FS}$ = fator de segurança, considerado como 10 (Brouwer et al., 1990).

O critério utilizado para classificar a segurança das condições de trabalho em função do valor de MS, conforme Machado Neto (1997), foi o seguinte: se MS calculada for maior ou igual a $1(\mathrm{MS} \geq 1)$, a condição foi classificada como segura; a exposição, aceitável; e o risco de intoxicação, tolerável. Nessas condições, a quantidade absorvivel da exposição multiplicada pelo fator de segurança 10 é menor que a dose segura (NOEL x $70 \mathrm{~kg}$ ). Se MS $<1$, a condição de trabalho foi classificada como insegura; a exposição, inaceitável; e o risco de intoxicação, intolerável. Nessas condições, a quantidade absorvivel da exposição, multiplicada pelo fator de segurança 10 , é maior que a dose segura.

\section{RESULTADOS E DISCUSSÃO}

\section{Exposições dérmicas e respiratórias potenciais às caldas e eficiência das medidas de segurança testadas}

Os resultados das exposições dérmicas e respiratórias (EDs e ERs) dos tratoristas às caldas, proporcionadas pelas oito condições de trabalho de aplicação de herbicida na cultura de cana-de-açúcar avaliada, estão apresentados na Tabela 1. Em todas as condições de trabalho, a principal via de exposição foi a dérmica, e a respiratória foi desprezivel, menor que $0,001 \%$ do total. Esses resultados concordam com os de Machado Neto (1997), Oliveira (2000), Cristóforo (2003), Oliveira (2004) e Tácio (2005). As ERs avaliadas nesses trabalhos representaram, no máximo, 0,040\% das exposições totais, embora tenham sido ligeiramente superiores às avaliadas no presente trabalho.

Das oito condições de trabalho avaliadas, a que proporcionou a maior exposição dérmica do tratorista $\left(65,4 \mathrm{~mL}\right.$ de calda dia $\left.^{-1}\right)$ foi a com o pulverizador de barra traseira montado no

Tabela 1 - Valores médios das exposições dérmicas (EDs) e exposições respiratórias (ERs) proporcionadas ao tratorista na pulverização de herbicidas em cultura de cana-de-açúcar com pulverizador de barra, nas oito condições de trabalho avaliadas. Jaboticabal-SP, 2006

\begin{tabular}{|c|c|c|c|c|}
\hline \multirow{2}{*}{ Condições de trabalho } & \multicolumn{4}{|c|}{ Exposições (mL de calda dib)** } \\
\cline { 2 - 5 } & $\mathrm{ED}^{*}$ & $\mathrm{DP}^{\mathrm{I}}$ & $\mathrm{ER}^{*}$ & Total \\
\hline $\begin{array}{c}\text { 1. Barra central trator } \\
\text { sem cabina ponta TF }\end{array}$ & 41,3 & 11,9 & 0,00 & 41,3 \\
\hline $\begin{array}{c}\text { 2. Barra central trator } \\
\text { sem cabina ponta TTI }\end{array}$ & 36,9 & 13,1 & 0,00 & 36,9 \\
\hline $\begin{array}{c}\text { 3. Barra central trator } \\
\text { com cabina ponta TF }\end{array}$ & 26,9 & 5,5 & 0,00 & 26,9 \\
\hline $\begin{array}{c}\text { 4. Barra central trator } \\
\text { com cabina ponta TTI }\end{array}$ & 19,2 & 3,8 & 0,00 & 19,2 \\
\hline $\begin{array}{c}\text { 5. Barra traseira trator } \\
\text { sem cabina ponta TF }\end{array}$ & 52,9 & 10,8 & 0,00 & 52,9 \\
\hline $\begin{array}{c}\text { 6. Barra traseira trator } \\
\text { sem cabina ponta TTI }\end{array}$ & 65,4 & 23,8 & 0,00 & 65,4 \\
\hline $\begin{array}{c}\text { 7. Barra traseira trator } \\
\text { com cabina ponta TF }\end{array}$ & 53,8 & 6,4 & 0,00 & 53,8 \\
\hline $\begin{array}{c}\text { 8. Barra traseira trator } \\
\text { com cabina ponta TTI }\end{array}$ & 55,0 & 9,7 & 0,00 & 55,0 \\
\hline
\end{tabular}

* ED - exposição dérmica; ER - exposição respiratória; ** um dia de trabalho de oito horas; $\stackrel{1}{ }$ desvio-padrão da média.

Planta Daninha, Viçosa-MG v. 25, n. 3, p. 639-648, 2007 
trator sem cabina e utilizando as pontas TTI (com indução de ar). A menor ED do tratorista $\left(19,2 \mathrm{~mL}\right.$ de calda dia $\left.{ }^{-1}\right)$ ocorreu com o pulverizador com a barra central montado no trator com cabina, utilizando as pontas TTI. Essas diferenças devem-se aos efeitos da posição central da barra do pulverizador e da cabina do trator atuando na redução da exposição dérmica proporcionada pela condição de trabalho ao tratorista. Esses efeitos dessas alterações estruturais no pulverizador as caracterizam como medidas de proteção coletiva, de acordo com Machado Neto \& Matuo (1992), Machado Neto et al. (1992) e ANVISA (2006). As EDs das demais condições de trabalho, avaliadas com as demais combinações dos fatores estudados, foram intermediárias a estas.

Os resultados das análises estatísticas dos dados de exposição dérmica estão apresentados na Tabela 2. O coeficiente de variação da análise de variância foi de $27,4 \%$. Esse valor é

Tabela 2 - Resumo das análises estatísticas dos dados de exposições dérmicas às caldas proporcionadas ao tratorista, nas oito condições de trabalho, na aplicação de herbicidas na cultura de cana-de-açúcar com pulverizador de barra. Jaboticabal-SP, 2006

\begin{tabular}{|c|c|}
\hline Causas da variação & Valores de $\mathrm{F}$ \\
\hline Posição da barra (PB) & $95,07 * *$ \\
\hline Cabina (CB) & $13,19 * *$ \\
\hline Modelo da ponta (MP) & $0,00^{\mathrm{ns}}$ \\
\hline $\mathrm{PB} \times \mathrm{CB}$ & $5,18 *$ \\
\hline PB $\times$ MP & $4,94 *$ \\
\hline $\mathrm{CB} \times \mathrm{MP}$ & $2,42^{\mathrm{ns}}$ \\
\hline $\mathrm{PB} \times \mathrm{CB} \times \mathrm{MP}$ & $0,90^{\mathrm{ns}}$ \\
\hline $\mathrm{CV}(\%)$ & 27,41 \\
\hline Posição da barra & mL de calda dia ${ }^{-1}$ \\
\hline Traseira & $57,53 \mathrm{~A}^{\mathrm{U}}$ \\
\hline Central & $31,05 \mathrm{~B}$ \\
\hline DMS (5\%) & 5,41 \\
\hline Cabina & mL de calda dia ${ }^{-1}$ \\
\hline Sem cabina & $49,22 \mathrm{~A}$ \\
\hline Com cabina & $39,36 \mathrm{~B}$ \\
\hline DMS (5\%) & 5,41 \\
\hline Modelo da ponta & mL de calda dia $^{-1}$ \\
\hline Sem indução de ar (TF) & $44,30 \mathrm{~A}$ \\
\hline Com indução de ar (TTI) & $44,27 \mathrm{~A}$ \\
\hline DMS (5\%) & 5,41 \\
\hline
\end{tabular}

* e ** e ns - significativos a 1 e $5 \%$ e não-significativo, respectivamente.

${ }^{\text {}}$ Médias seguidas por mesma letra na coluna e dentro do mesmo fator não diferem entre si pelo teste de Tukey $(\mathrm{P}>0,05)$. muito inferior aos $70,2 \%$ calculados por Momesso \& Machado Neto (2002) para o tratorista em aplicação de herbicidas também em cultura de cana-de-açúcar. Esses valores de coeficientes de variação estão dentro das variações aceitáveis para o coeficiente de variação nesse tipo de trabalho.

Verifica-se que houve efeitos significativos dos fatores posição da barra e uso da cabina do trator e não-significativo do modelo de ponta de pulverização. A barra central, em média, proporcionou exposição dérmica ao tratorista significativamente menor $(46,1 \%)$ que a barra traseira. Não foram encontrados outros trabalhos de pesquisa que possam ser comparados com esses resultados. Entretanto, essa menor exposição deve-se à posição do tratorista acima e mais perto das secções laterais da barra e mais longe e atrás da estrutura frontal do trator da secção central da barra, de comprimento do rodado do trator, afixada no párachoque à frente do trator, em relação à posição da barra traseira.

O uso da cabina no trator, em média, proporcionou exposição dérmica ao tratorista significativamente menor $(20,0 \%)$ que aquela com o trator sem cabina. Esse resultado é inferior aos obtidos por outros autores, que utilizaram outros tipos de pontas de pulverização. A cabina do trator proporcionou reduções das EDs do tratorista entre 38,5 e $77,0 \%$ (Momesso \& Machado Neto, 2003) e entre 95,8 e 87,8\% (Cristóforo, 2003). A menor eficiência no controle da exposição dos tratoristas pela cabina do trator (Tabela 2) pode ser explicada pelas baixas exposições proporcionadas pelas condições de trabalho aos tratoristas. De maneira geral, constata-se a importância da cabina do trator como medida de segurança coletiva em aplicações de herbicidas em cana-de-açúcar com o pulverizador de barra (Momesso \& Machado Neto, 2002; Machado Neto \& Oliveira, 2006), e de inseticidas, fungicidas e acaricidas com o turbopulverizador em cultura de citros (Oliveira, 2000) e goiaba (Tácio, 2005).

Os dois tipos de pontas de pulverização não causaram diferenças significativas nas EDs dos tratoristas. A proximidade nos valores das exposicões proporcionadas pelas pontas de pulverização pode ser explicada pela semelhança entre o tamanho das gotas (DMV) e a 
uniformidade do espectro (span). As gotas produzidas por ambas as pontas classificamse na categoria de extremamente grossas, que proporcionam baixas derivas e evaporação (Gadanha Junior, 2000; Wolf, 2000a, b; Boller, 2004).

Na Tabela 3 estão apresentados os desdobramentos estatísticos dos efeitos de cada fator dentro dos demais, para controle da exposição dérmica dos tratoristas. Com a barra central, a exposição dérmica do tratorista foi reduzida em $28,3 \%$ com as pontas $\mathrm{TF}$ sem a cabina e em $100,3 \%$ associada com o uso da cabina. Com a barra central e as pontas TTI e sem a cabina do trator, a eficiência foi de $77,5 \%$ de controle da exposição dérmica do tratorista e de $186,9 \%$ com a cabina. Nessas condições específicas de trabalho, o uso da barra central proporcionou controles da exposição dérmica do tratorista superiores à média de 45,6\%, em relação ao uso da barra traseira (Tabela 2), exceto na condição de trabalho com a ponta TF sem a cabina do trator. Não foram encontrados trabalhos publicados sobre os efeitos dos fatores ora estudados na exposição dérmica do tratorista.

A eficiência da cabina do trator com medida de proteção coletiva foi maior quando associada à barra central, cuja exposição dérmica do tratorista foi reduzida em $34,9 \%$ com o uso das pontas TF e em 47,9\% com as pontas TTI.
A eficiência do uso da cabina no trator associada à barra traseira foi nula com as pontas TF e reduziu em apenas 15,9\% a exposição do tratorista com as pontas TTI. As baixas eficiências da cabina do trator avaliadas neste trabalho foram devidas às baixas exposições potenciais proporcionadas ao tratorista pelas condições de trabalho avaliadas.

Para o fator ponta de pulverização (Tabela 3), com a barra central, a ponta TTI proporcionou controle da exposição de $10,7 \%$ sem a cabina e de $28,7 \%$ com a cabina. Com a barra traseira, a ponta TTI não proporcionou controle da exposição dérmica do tratorista. Esta ponta foi mais segura quando utilizada nos pulverizadores de barra central e no trator com cabina.

\section{Segurança das condições de trabalho}

Os valores das margens de segurança (MSs) calculados para os tratoristas, nas oito condições de trabalho avaliadas, estão apresentados na Tabela 4. A condição mais segura para o tratorista ocorreu com o pulverizador de barra central, trator com cabina e pontas TTI, devido à menor exposição dérmica proporcionada ao tratorista. Das 46 recomendações consideradas, 15 classificaram-se como seguras ( $\mathrm{MS} \geq 1$ ) para o tratorista com o trator sem cabina, com a barra central e com os dois tipos de pontas: sem indução de ar (TF) e com indução

Tabela 3 - Eficiência das medidas de proteção ao tratorista, nas oito condições de pulverização de herbicidas, na cultura de cana-deaçúcar com pulverizad ores de barra. Jaboticabal-SP, 2006

\begin{tabular}{|c|l|c|}
\hline Medida de proteção avaliada & Condições do pulverizador & Controle da exposição potencial (\%) \\
\hline \multirow{2}{*}{$\begin{array}{c}\text { Barra Central } \\
\text { X }\end{array}$} & Ponta TF sem Cabina & 28,3 \\
\cline { 2 - 3 } Barra Traseira (testemunha*) & Ponta TF com Cabina & 100,3 \\
\cline { 2 - 3 } & Ponta TTI sem Cabina & 17,5 \\
\cline { 2 - 3 } Cabina & Ponta TTI com Cabina & 34,9 \\
\hline \multirow{3}{*}{$\begin{array}{c}\text { Xem Cabina (testemunha*) } \\
\text { Ponta TTI } \\
\text { X }\end{array}$} & Barra Central com Ponta TF & 47,9 \\
\cline { 2 - 3 } & Barra Central com Ponta TTI & - \\
\cline { 2 - 3 } & Barra Traseira com Ponta TF & 15,9 \\
\cline { 2 - 3 } & Barra Traseira com Ponta TTI & 10,7 \\
\hline Ponta TF (testemunha*) & Barra Central sem Cabina & 28,7 \\
\cline { 2 - 3 } & Barra Central com Cabina & - \\
\cline { 2 - 3 } & Barra Traseira sem Cabina & - \\
\cline { 2 - 3 } & Barra Traseira com Cabina & \\
\hline
\end{tabular}

* testemunha: exposição potencial considerada como $100 \%$. 
Tabela 4 - Valores da margem de segurança (MS) calculados para o tratorista nas recomendações dos herbicidas registrados para a cultura de cana-de-açúcar, na maior dosagem, com volume de $200 \mathrm{~L} \mathrm{ha}^{-1}$. Jaboticabal-SP, 2006

\begin{tabular}{|c|c|c|c|c|c|c|c|c|c|}
\hline \multirow{3}{*}{ Ingrediente ativo } & \multirow{3}{*}{$\begin{array}{l}\text { mg i.a./mL } \\
\text { de calda }\end{array}$} & \multicolumn{4}{|c|}{ Barra central } & \multicolumn{4}{|c|}{ Barra traseira } \\
\hline & & \multicolumn{2}{|c|}{ Ponta s indução ar } & \multicolumn{2}{|c|}{ Ponta c/indução ar } & \multicolumn{2}{|c|}{ Ponta s/indução ar } & \multicolumn{2}{|c|}{ Ponta c/indução ar } \\
\hline & & $\begin{array}{l}\text { MS s/ } \\
\text { cabina }\end{array}$ & $\begin{array}{c}\text { MS } \\
\text { c/cabina }\end{array}$ & $\begin{array}{c}\text { MS } \\
\text { s/cabina }\end{array}$ & $\begin{array}{c}\text { MS } \\
\text { c/cabina }\end{array}$ & $\begin{array}{c}\text { MS } \\
\text { s/cabina }\end{array}$ & $\begin{array}{c}\text { MS } \\
\text { c/cabina }\end{array}$ & $\begin{array}{c}\text { MS } \\
\text { s/cabina }\end{array}$ & $\begin{array}{c}\text { MS } \\
\text { c/cabina }\end{array}$ \\
\hline Imazapyr & 2,66 & 159,3 & 244,7 & 178,4 & 343,2 & 124,2 & 122,2 & 100,5 & 119,6 \\
\hline Trifloxysulfuron-sodium & 0,19 & 137,2 & 210,7 & 153,8 & 295,5 & 107,2 & 105,4 & 86,7 & 103,2 \\
\hline Imazapic & 1,86 & 125,2 & 192,2 & 140,2 & 269,6 & 97,6 & 96,0 & 79,0 & 94,0 \\
\hline Glyphosate & 9,00 & 17,7 & 27,1 & 19,8 & 38,0 & 13,8 & 13,5 & 11,1 & 13,3 \\
\hline Amicarbazone & 5,25 & 12,9 & 19,8 & 14,5 & 27,8 & 10,1 & 9,9 & 8,2 & 9,7 \\
\hline Hexazinone & 3,13 & 5,4 & 8,3 & 6,1 & 11,7 & 4,2 & 4,2 & 3,4 & 4,1 \\
\hline Sulfentrazone & 5,00 & 3,4 & 5,2 & 3,8 & 7,3 & 2,6 & 2,6 & 2,1 & 2,5 \\
\hline Clomazone & 7,50 & 3,2 & 4,9 & 3,5 & 6,8 & 2,5 & 2,4 & 2,0 & 2,4 \\
\hline Oxadiazon & 3,13 & 2,7 & 4,2 & 3,0 & 5,8 & 2,1 & 2,1 & 1,7 & 2,0 \\
\hline Isoxaflutole & 1,31 & 2,6 & 4,0 & 2,9 & 5,6 & 2,0 & 2,0 & 1,6 & 1,9 \\
\hline Flazasulfurom & 0,50 & 2,1 & 3,3 & 2,4 & 4,6 & 1,7 & 1,6 & 1,3 & 1,6 \\
\hline Pendimethalin & 8,75 & 2,3 & 3,6 & 2,6 & 5,0 & 1,8 & 1,8 & 1,5 & 1,7 \\
\hline Tebuthiuron & 6,00 & 2,0 & 3,0 & 2,2 & 4,3 & 1,5 & 1,5 & 1,2 & 1,5 \\
\hline Ethoxysulfuron & 0,75 & 1,4 & 2,2 & 1,6 & 3,0 & 1,1 & 1,1 & 0,9 & 1,1 \\
\hline Acetoclor & 16,80 & 1,0 & 1,5 & 1,1 & 2,2 & 0,8 & 0,8 & 0,6 & 0,8 \\
\hline S-metalochlor & 14,40 & 0,9 & 1,4 & 1,0 & 1,9 & 0,7 & 0,7 & 0,6 & 0,7 \\
\hline Oxifluorfen & 6,00 & 0,7 & 1,1 & 0,8 & 1,5 & 0,6 & 0,5 & 0,4 & 0,5 \\
\hline Metribuzin & 12,00 & 0,5 & 0,7 & 0,5 & 1,0 & 0,4 & 0,4 & 0,3 & 0,4 \\
\hline Alachlor & 16,80 & 0,1 & 0,1 & 0,1 & 0,1 & 0,0 & 0,0 & 0,0 & 0,0 \\
\hline Asulam & 18,00 & 0,0 & 0,1 & 0,1 & 0,1 & 0,0 & 0,0 & 0,0 & 0,0 \\
\hline Ametryn & 20,00 & 0,2 & 0,3 & 0,2 & 0,4 & 0,1 & 0,1 & 0,1 & 0,1 \\
\hline Atrazine & 17,50 & 0,0 & 0,1 & 0,1 & 0,1 & 0,0 & 0,0 & 0,0 & 0,0 \\
\hline Azafenidin & 10,00 & 0,0 & 0,0 & 0,0 & 0,0 & 0,0 & 0,0 & 0,0 & 0,0 \\
\hline Cyanazine & 12,50 & 0,0 & 0,0 & 0,0 & 0,1 & 0,0 & 0,0 & 0,0 & 0,0 \\
\hline Paraquat & 3,60 & 0,2 & 0,3 & 0,2 & 0,4 & 0,1 & 0,1 & 0,1 & 0,1 \\
\hline Diuron & 9,36 & 0,1 & 0,2 & 0,1 & 0,2 & 0,1 & 0,1 & 0,1 & 0,1 \\
\hline Fluazifop-p-butyl & 1,25 & 0,4 & 0,6 & 0,5 & 0,9 & 0,3 & 0,3 & 0,3 & 0,3 \\
\hline Halosulfuron-metyl & 7,50 & 0,2 & 0,3 & 0,3 & 0,5 & 0,2 & 0,2 & 0,1 & 0,2 \\
\hline Msma & 10,80 & 0,1 & 0,1 & 0,1 & 0,2 & 0,1 & 0,1 & 0,0 & 0,1 \\
\hline Simazine & 12,50 & 0,1 & 0,1 & 0,1 & 0,1 & 0,1 & 0,1 & 0,0 & 0,1 \\
\hline Sulfosate & 14,40 & 0,0 & 0,0 & 0,0 & 0,0 & 0,0 & 0,0 & 0,0 & 0,0 \\
\hline \multirow{2}{*}{ Tiazopir Trifluralin } & 1,80 & 0,0 & 0,0 & 0,0 & 0,0 & 0,0 & 0,0 & 0,0 & 0,0 \\
\hline & 12,00 & 0,4 & 0,5 & 0,4 & 0,8 & 0,3 & 0,3 & 0,2 & 0,3 \\
\hline $2,4-\mathrm{D}$ & 12,09 & 0,1 & 0,2 & 0,2 & 0,3 & 0,1 & 0,1 & 0,1 & 0,1 \\
\hline \multirow{2}{*}{ Ametryn + Clomazone } & 12,00 & 0,3 & 0,4 & 0,3 & 0,6 & 0,2 & 0,2 & 0,2 & 0,2 \\
\hline & 6,00 & 4,0 & 6,1 & 4,4 & 8,5 & 3,1 & 3,0 & 2,5 & 3,0 \\
\hline \multirow{2}{*}{$\begin{array}{l}\text { Ametryn + } \\
\text { Trifloxysulfuron-sodium }\end{array}$} & 7,33 & 0,5 & 0,7 & 0,5 & 1,0 & 0,4 & 0,4 & 0,3 & 0,3 \\
\hline & 0,19 & 137,2 & 210,7 & 153,8 & 295,5 & 107,2 & 105,4 & 86,7 & 103,2 \\
\hline \multirow{2}{*}{ Azafenidin + Hexazinone } & 0,61 & 0,1 & 0,2 & 0,1 & 0,2 & 0,1 & 0,1 & 0,1 & 0,1 \\
\hline & 0,79 & 21,5 & 33,0 & 24,1 & 46,4 & 16,8 & 16,5 & 13,6 & 16,2 \\
\hline \multirow{2}{*}{ Diuron + hexazinone } & 7,02 & 0,2 & 0,2 & 0,2 & 0,3 & 0,1 & 0,1 & 0,1 & 0,1 \\
\hline & 1,98 & 8,6 & 13,1 & 9,6 & 18,4 & 6,7 & 6,6 & 5,4 & 6,4 \\
\hline \multirow{2}{*}{ 2,4-D + Picloran } & 8,70 & 0,2 & 0,3 & 0,2 & 0,4 & 0,2 & 0,1 & 0,1 & 0,1 \\
\hline & 0,53 & 22,3 & 34,2 & 25,0 & 48,0 & 17,4 & 17,1 & 14,1 & 16,7 \\
\hline & 1,50 & 0,7 & 1,1 & 0,8 & 1,5 & 0,6 & 0,5 & 0,4 & 0,5 \\
\hline Diuron + Paraquat & 3,00 & 0,2 & 0,3 & 0,2 & 0,5 & 0,2 & 0,2 & 0,1 & 0,2 \\
\hline Alachlor + Atrazine & 9,60 & 0,1 & 0,1 & 0,1 & 0,2 & 0,1 & 0,1 & 0,0 & 0,1 \\
\hline Alacnior + Aurazine & 10,00 & 0,1 & 0,2 & 0,1 & 0,3 & 0,1 & 0,1 & 0,1 & 0,1 \\
\hline Ametryn + Diuron & 9,60 & 0,4 & 0,5 & 0,4 & 0,8 & 0,3 & 0,3 & 0,2 & 0,3 \\
\hline Ametryn + Diuron & 6,20 & 0,2 & 0,3 & 0,2 & 0,4 & 0,1 & 0,1 & 0,1 & 0,1 \\
\hline Ametryn + Simazine & 10,00 & 0,3 & 0,5 & 0,4 & 0,7 & 0,3 & 0,3 & 0,2 & 0,3 \\
\hline Amенуи + sminazme & 10,00 & 0,1 & 0,1 & 0,1 & 0,2 & 0,1 & 0,1 & 0,1 & 0,1 \\
\hline Atrazine + Simazine & 6,88 & 0,1 & 0,2 & 0,1 & 0,3 & 0,1 & 0,1 & 0,1 & 0,1 \\
\hline Atrazıne + simazine & 6,88 & 0,1 & 0,2 & 0,1 & 0,3 & 0,1 & 0,1 & 0,1 & 0,1 \\
\hline & 7,00 & 0,2 & 0,2 & 0,2 & 0,3 & 0,1 & 0,1 & 0,1 & 0,1 \\
\hline Diuron + msma & 18,00 & 0,1 & 0,1 & 0,1 & 0,1 & 0,0 & 0,0 & 0,0 & 0,0 \\
\hline tehuthiuron & 10,00 & 0,1 & 0,2 & 0,1 & 0,2 & 0,1 & 0,1 & 0,1 & 0,1 \\
\hline Diuron + tebuthiuron & 4,00 & 0,3 & 0,4 & 0,3 & 0,6 & 0,2 & 0,2 & 0,2 & 0,2 \\
\hline
\end{tabular}

Seguras $(M S \geq 1)$ em negrito e inseguras (MS $<1$ ) em itálico. 
de ar (TTI). Nas quatro condições de trabalho, em ordem decrescente de valor da MS, foram seguras as pulverizações dos herbicidas: imazapyr, trifloxysulfuron-sodium, imazapic, glyphosate, amicarbazone, hexazinone, sulfentrazone, clomazone, oxadiazon, isoxaflutole, pendimethalin, flazasulfuron, tebuthiuron, ethoxysulfuron e acetoclor. Para essas 15 recomendações de herbicidas que se classificaram como seguras, cujos valores de TTS calculados foram superiores aos da jornada considerada de oito horas de trabalho, outras medidas de segurança poderiam ser recomendadas como medidas preventivas de exposições acidentais - por exemplo, uso da cabina no trator e de equipamentos de proteção individual.

Com o uso da cabina no trator, como medida de proteção coletiva, tornaram-se seguras apenas as pulverizações dos herbicidas oxifluorfen e s-metalochlor. Esses resultados diferem dos obtidos por Momesso \& Machado Neto (2003), em que as aplicações de um número maior de herbicidas foram seguras. Essa diferença pode ser devida à maior exposição dérmica proporcionada ao tratorista no presente trabalho, em relação à avaliada por Momesso $\&$ Machado Neto (2003).

Quanto à toxicidade dos herbicidas, verifica-se que os mais tóxicos, com menor valor de NOEL, para a mesma condição de uso e dose, proporcionaram condição de trabalho insegura (MS < 1), e vice-versa. Por sua vez, as formulações com misturas de herbicidas também são afetadas pela toxicidade de cada um dos herbicidas presentes. A segurança da condição de trabalho é baseada no menor valor de MS calculado para cada ingrediente ativo. Por exemplo, a formulação composta pelos herbicidas diuron + paraquat foi classificada como insegura devido ao paraquat $(\mathrm{MS}<1)$ e não ao diuron (MS > 1).

Nas condições de trabalho com a barra traseira e o trator sem cabina, 14 das 46 recomendações de herbicidas classificaram-se como seguras para o tratorista com as pontas TF e 13 com as pontas TTI. Na condição de trabalho com as pontas TTI, em ordem decrescente de valor da MS, foram seguras as pulverizações dos herbicidas: imazapyr, trifloxys ulfuron-sodium, imaza pic, gly phosate, amicarbazone, hexazinone, sulfen trazone, clomazone, oxadiazon, isoxaflutole, pendimethalin, flazasulfuron e tebuthiuron. Na condição de trabalho com as pontas TF, em ordem decrescente de intensidade, foram seguras as pulverizações de todos os herbicidas com as pontas TTI e mais o ethoxysulfuron.

Pelos resultados deste trabalho, o uso da barra central do pulverizador no trator é uma medida de proteção coletiva eficiente para o tratorista em pulverizações de herbicidas em pré e pós-emergência da cana-de-açúcar quando associado ao uso da cabina do trator. A cabina no trator é uma medida de proteção coletiva eficiente para o tratorista em aplicações de herbicidas em pré e pós-emergência na canade-açúcar com pulverizadores de barra central ou traseira.

\section{LITERATURA CITADA}

AGÊNCIA NACIONAL DE VIGILÂNCIA SANITÁRIA ANVISA. Resolução n ${ }^{\circ}$ 2606, de 11- 08 - 2006. Diário Oficial da União, Poder Executivo, Brasília, 14 ago 2006. Disponível em: $<$ http://e-legis.anvisa.gov.br/leisref/public/ showAct.php?id=235 98word= >. Acesso em: 21 maio 2007.

ANDREI, E. Compêndio de defensivos agrícolas: guia prático de produtos fitossanitários para uso agrícola. 6.ed. São Paulo: Organização Andrei, 2003. 672 p.

BONSALL, J. L. Measurement of occupational exposure to pesticide. In: TURNBULL, G. L. (Ed.). Occupational hazards of pesticide use. London: Taylor \& Francis, 1985. p. 13-23.

COUTINHO, P. O.; CORDEIRO, C. A. M.; MOTTA, F. Princípios gerais da tecnologia de aplicação. In: DUPONT DO BRASIL. Tecnologia de Aplicação de Defensivos. São Paulo Editora: Boletim Técnico DuPont - Tecnologias Integradas Soluções Técnicas para a Agricultura, 2003. p. 4-6.

CRISTÓFORO, A. B. Segurança das condições de aplicação de agrotóxicos em cultura de soja e amendoim e eficiência de medidas de segurança individual e coletiva. 2003. 60 f. Dissertação (Mestrado em Agronomia Área de Concentração em Entomologia Agrícola) Universidade Estadual Paulista, Jaboticabal, 2003.

GADANHA JUNIOR, C. D. O desenvolvimento de produtos, equipamentos e componentes na aplicação de agrotóxicos, tendências e realidade: evolução dos equipamentos de aplicação. In: SIMPÓSIO INTERNACIONAL DE TECNOLOGIA DE APLICAÇÃO DE AGROTÓXICOS: EFICIÊNCIA, ECONOMIA E PRESERVAÇÃO DA SAÚDE HUMANA E DO MEIO AMBIENTE, 2., 2000, Jundiaí. Anais... Jundiaí: 2000. 
HIMEL, C. M. The optimum size for insecticide spray droplets. J. Econ. Entomol., v. 62, n. 4, p. 919-925, 1969.

JENSEN, J. K. The assumptions used for exposure assessments. In: SIEWIERSKI, M. (Ed.). Determination and assessment of pesticide exposure. New York: Elsevier, 1984. p. 147-152.

MACHADO NETO, J. G. Segurança no trabalho com agrotóxicos em cultura de eucalipto. Jaboticabal: Funep, 2001. $105 \mathrm{p}$.

MACHADO NETO, J. G.; MATUO, T. Avaliação de um amostrador para estudo da exposição dérmica de aplicadores de defensivos agrícolas. Ci. Agron., v. 4, n. 2, p. 22, 1989.

MACHADO NETO, J. G.; MATUO, T. Safety of hand operated knapsack sprayers in the application of paraquat on corn (Zea mays L.) by small scale farmers. In: INTERNATIONAL SCIENTIFIC CONFERENCE ON OCCUPATIONAL HYGIENE, 1., 1992, Bussels. Book of abstracts Workshop C., Bussels: 1992.

MACHADO NETO, J. G.; MATUO, T.; MATUO, Y. K. Dermal exposure of pesticide applicators in staked tomato (Lycopersicon esculentum) crops: efficiency of safety measure in the application equipament. B. Environ. Contam. Toxicol., v. 48, p. 529-534, 1992.

MACHADO NETO, J. G.; MATUO, T.; MATUO, Y. K. Exposição dérmica de aplicadores de agrotóxicos na cultura estaqueada de tomate (Lycopersicon esculentum Mill.) eficiência de equipamentos de proteção individual. R. Bras. Saúde Ocupacional, v. 21, n. 79, p. 29-38, 1993.

MACHADO NETO, J. G.; OLIVEIRA, M. L. Segurança no trabalho com herbicidas aplicados com o pulverizad or de barra em cultura de cana-de-açúcar. In: CONGRESSO BRASILEIRO DE PLANTAS DANINHAS, 2006, Brasília. Anais... Brasília: 2006. CD-ROM.

MACHADO-NETO, J. G. Estimativas do tempo de trabalho seguro e da necessidade de controle da exposição dos aplicadores de agrotóxicos. 1997. $83 \mathrm{f}$. Tese (Livre-Docência) - Universidade Estadual Paulista, Jaboticabal, 1997.

MOMESSO, J. C.; MACHADO NETO, J. G. Efeito do período e do volume de aplicação na segurança dos tratoristas aplicando herbicida na cultura de cana-de-açúcar (Saccharum spp.) Planta Daninha, v. 21, n. 3, p. 467-478, 2003.

OLIVEIRA, M. L. Avaliação e incremento na segurança do trabalho de aplicação de agrotóxicos em cultura de batata e permeabilidade de dois tipos de tecido de algodão ao metamidophos. 2004. 93 f. Tese (Doutorado em Agronomia - Produção Vegetal) - Universidade Estadual Paulista, Jaboticabal, 2004.
OLIVEIRA, M. L. Segurança no trabalho de aplicação de agrotóxicos com turboatomizador e pulverizador de pistolas em citros. 2000. 99 f. Dissertação (Mestrado em Agronomia - Produção Vegetal) - Universidade Estadual Paulista, Jaboticabal, 2000.

OLIVEIRA, M. L.; MACHADO NETO, J. G. Segurança no trabalho de aplicação de agrotóxicos com o pulverizador de pistolas em citros. R. Bras. Saúde Ocupacional, v. 28, n. $105 / 106$, p. $83-90,2003 b$.

OLIVEIRA, M. L.; MACHADO NETO, J. G. Use of manganese as tracer in the determination of respiratory exposure and relative importance of exposure routes in safety of pesticide applicators in citrus orchards.

B. Environ. Contam. Toxicol., v. 70, n. 3, p. 415-421, 2003a.

PRADELA, V. A. Segurança dos aplicadores de glifosate em jato dirigido em cultura de cana-de-açúcar com pulverizadores costais. 1998. $36 \mathrm{f}$. Monografia (Trabalho de Engenharia de Segurança do Trabalho) - Centro

Universitário Moura Lacerda, Câmpus de Ribeirão Preto, Ribeirão Preto, 1998.

SISTEMAS DE INFORMAÇÃO SOBRE AGROTÓXICOS - SIA. Agência Nacional de Vigilância Sanitária, 2006. Disponível em: < http://www.anvisa.gov.br/ AGROSIA/ asp/frm >. Acesso em: 16 nov. 2006.

SINDICATO DA INDÚSTRIA DE PRODUTOS PARA DEFESA AGRÍCOLA - SINDAG. Vendas de defensivos agrícolas por cultura de destinação e classes, São Paulo. 2006.

SKOOG, D. A.; HOLLER, F. J.; NIEMAN, T. A. Princípios de análise instrumental. 5.ed. Porto Alegre: Bookman, 2002. $836 \mathrm{p}$.

SPRAYING SYSTEMS Co. Produtos para pulverização na agricultura. Wheaton: North Avenue at Schmale Road, 2006. $176 \mathrm{p}$.

TÁCIO, M. B. Segurança das condições de trabalho das pulverizações de agrotóxicos em cultura de goiaba no sistema de poda contínua. 2005. $49 \mathrm{f}$. (Trabalho de Graduação) - Universidade Estadual Paulista, Jaboticabal, 2005.

THERAPEUTIC GOODS ADMINISTRATION - TGA ADI LIST: acceptable daily intakes for agricultural and veterinary chemicals. Canberra, 2006. 34 p.

WORLD HEALTH ORGANIZATION - WHO. Field surveys of exposure to pesticide - standard protocol. Geneva, 1982. (Document, VBC/82.1). 